\title{
Transient Response and Fixed Pattern Noise in Logarithmic CMOS Image Sensors
}

\author{
Dileepan Joseph, Member, IEEE, and Steve Collins, Member, IEEE
}

\begin{abstract}
Logarithmic CMOS image sensors are appealing for their high-contrast and high-speed response but they require postprocessing to achieve high-quality images. Previously published work has explained the fixed pattern noise (FPN) in these image sensors using a steady-state analysis. This paper explains how the transient response of the readout circuit may also contribute to FPN. Thus, the performance of these CMOS cameras may be optimized with a proper understanding of the transient response, which is explained here through modeling and simulation with some experimental validation. In particular, the gain variation of a logarithmic camera is shown to be caused primarily by premature digitization. As logarithmic and linear active pixel sensors use similar circuits, some results in this paper, e.g., an analysis of readout capacitance, apply equally to the latter.
\end{abstract}

Index Terms-Fixed pattern noise (FPN), logarithmic pixels, modeling and calibration, transient response.

\section{INTRODUCTION}

$\mathbf{L}$ OGARITHMIC pixels are notorious for problems with image quality [1]. Nonetheless, they occupy a niche in the world of imaging because of their edge in dynamic range and bandwidth [2]. There is potential for this niche to grow, however, given the work of Joseph and Collins, who modeled the logarithmic pixel and employed calibration to achieve an image quality comparable to that of conventional linear pixels [3], [4]. Having proven the feasibility of achieving high-quality color images with these cameras, Collins et al. have since been migrating the approach towards self-calibration and have been reducing the computational load [5], [6]. This work has proven relevant to independent researchers, including some at the University of Edinburgh and the STMicroelectronics Imaging Division [7], [8].

Although the literature explains the steady-state response of logarithmic CMOS image sensors, showing how a pixel-to-pixel variation of device parameters causes fixed pattern noise (FPN), the previously published analysis neglects to explain the contribution of transient response to FPN. Since voltage changes need time for rising and falling, it is natural to expect that if

Manuscript received June 28, 2006; revised February 24, 2007; accepted March 1, 2007. This work was supported in part by the Natural Sciences and Engineering Research Council of Canada and in part by the Engineering and Physical Sciences Research Council of the United Kingdom. The associate editor coordinating the review of this paper and approving it for publication was Dr. Andre Bossche.

D. Joseph is with the Department of Electrical and Computer Engineering, University of Alberta, Edmonton, AB T6G 2V4, Canada (e-mail: dil.joseph@ualberta.ca).

S. Collins is with the Department of Engineering Science, University of Oxford, Oxford OX1 2JD, U.K. (e-mail: collins@ robots.ox.ac.uk).

Digital Object Identifier 10.1109/JSEN.2007.900974 insufficient time is provided then noise would also appear. Consequently, this paper explains how noise caused by improper timing displays a fixed pattern not explained in the literature.

A transient analysis of logarithmic imagers may include the response of the photodiode and the load transistor, which operates in weak inversion, but this is not done here for the following reasons. First, logarithmic pixels offer a high optical bandwidth because they operate continuously unlike linear integrating pixels. Using modulated lasers, Tabet et al. measured the small-signal $3 \mathrm{~dB}$ bandwidth to be $97.5 \mathrm{kHz}$ at 437 lux [9]. IMS Chips measured the large-signal settling time for a $10^{4}$-to- 1 lux transition to be $8 \mathrm{~ms}$ (a step change in the reverse direction settles in $0.8 \mu \mathrm{s}$ ) [10]. Note that logarithmic cameras are typically used in machine vision [2], often to automate a task performed by a human. A frame rate of $24-30 \mathrm{~Hz}$, i.e., a sampling period of 33-42 ms, satisfies the motion sensitivity of the human eye [11]. Therefore, when the light focused on a pixel is modulated by normal motion within a scene under normal lighting, the transient response of the photodiode and load transistor is fast enough to approximate the steady-state response. For applications where the logarithmic pixel proves too slow, only the variation in responsiveness from pixel-to-pixel contributes to FPN. This variation is unlikely to be as important as the slowness itself.

The transient response of the readout circuit, however, is crucial to the performance of the sensor. Unless there is space on the die for multiple analog-to-digital converters (ADCs), the pixel responses are raster scanned and digitized serially. In an array of $N_{1} \times N_{2}$ pixels, serial readout at a frame rate of $R$ requires a pixel scan rate of $N_{1} N_{2} R$, which would be around $100 \mathrm{MHz}$ for mega-pixel sensors operating at standard video rates. Given that switching the readout circuit from one pixel to another is necessarily a discontinous analog process, the transient behavior of the readout circuit may certainly contribute to noise in the resulting images. In addition, multiple-ADC sensors may suffer in the same way as single-ADC sensors when operated at the high video rates for which they were originally developed. Note that column-level data conversion has been used to obtain frame rates of $500-1000 \mathrm{~Hz}$ [12].

Although this paper examines the readout circuitry of $\log$ arithmic sensors, much of what is said applies also to linear CMOS sensors (but not to CCD sensors as their readout method is different). Section II models and simulates the transient response of the readout circuit. Section III describes how premature digitization causes FPN and how it may be partly accommodated by previously published methods of calibration. Section IV uses experimental results from a Fuga 15RGB logarithmic camera to demonstrate the modeled and simulated effects. 


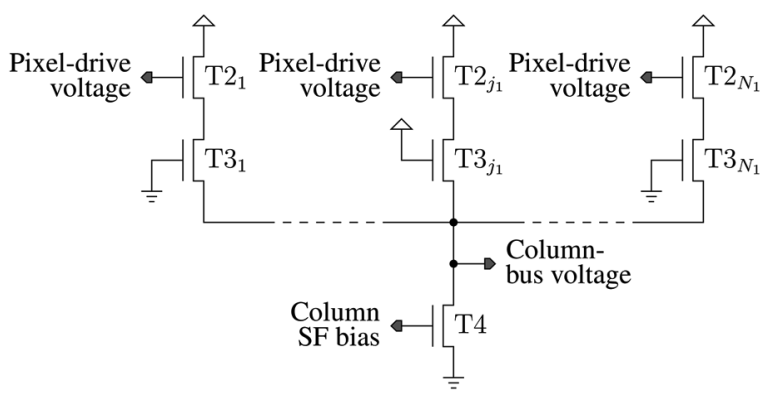

Fig. 1. The first-stage readout of a typical CMOS image sensor consists of $N_{1}$ amplifier and switch transistors T2 and T3 (one pair in each pixel of a column) and a current source T4 (at the base of the column). When switch $\mathrm{T} 3_{j_{1}}$ is on, where $1 \leq j_{1} \leq N_{1}$, all other switches are off and $\mathrm{T} 2_{j_{1}}$ forms a source follower (SF) with $\mathrm{T} 4$. The second-stage readout is similar but uses PMOS instead of NMOS transistors [3].

\section{TRANSIENT RESPONSE}

The digital response $y$ of a logarithmic pixel depends on the light stimulus $x$ by the model

$$
y=a+b \ln (c+x)+\epsilon
$$

where $a, b, c$, and $\epsilon$ are called the offset, gain, bias, and error, respectively [3]. While $\epsilon$ may vary randomly from sample to sample, $a, b$, and $c$ are constant for a pixel. One arrives at the "triple variation" model of FPN in a logarithmic camera by assuming the offset, gain, and bias are free to vary from one pixel to another, and that the error follows a zero-mean normal distribution. Although this model and an associated method of calibration have been validated, the model fails to explain spatial patterns found in estimated parameters for a commercial camera, as will be seen in Section IV. These patterns change when the frame rate is changed despite a static calibration scene, which suggests a contribution to FPN from the transient response of the readout circuit. Therefore, the established steadystate analysis is extended here to include the latter.

To read a frame, pixel responses are raster-scanned. A row is selected and the responses of all pixels in that row are copied to $N_{2}$ parallel buffers, one for each column. This is the first stage of readout. Each column, one of which is depicted in Fig. 1, consists of $N_{1}$ pixels connected to a common bus via $N_{1}$ source followers that share a current source, i.e., transistor T4, but have separate amplifiers, e.g., transistor $\mathrm{T} 2 j_{1}$ for pixel $j_{1}$.

As each pixel circuit also has a switch, e.g., transistor $\mathrm{T} 3_{j_{1}}$ for pixel $j_{1}$, the source follower may be operated in sequence for each pixel by closing the switch for that pixel and opening the switches of all other pixels, as shown in the figure for pixel $j_{1}$. In this manner, the column-bus voltage, denoted $V_{D}^{\mathrm{T}} 4$, follows the pixel-drive voltage, denoted $V_{G}^{\mathrm{T} 2}{ }_{j_{1}}$. The column-bus and pixel-drive voltages are the source-follower output and input, respectively. In the second stage of readout, assuming chip-level ADC, each column buffer is selected in sequence and copied to an output buffer that serves a data converter.

To perform a transient analysis, assume that no more than one switch is on at a time in the circuit of Fig. 1 and that the switches behave in an ideal fashion except for their capacitance, as described below. An expression may be derived, as a function of time $t$, for the column-bus voltage $v_{D}^{\mathrm{T}} 4(t)$ in terms of

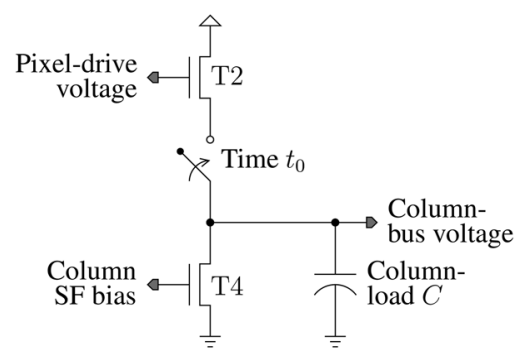

Fig. 2. The transient response of the first-stage readout for a pixel-drive voltage may be derived by analyzing a two-transistor source follower (SF), formed by $\mathrm{T} 2$ and T4 when only one switch is turned on in the circuit of Fig. 1, with a load capacitance $C$. When the switch is turned on at time $t_{0}$, the column bus may have a nonzero voltage due to readout of the previous pixel in the column or a zero voltage due to discharge.

a constant pixel-drive voltage $V_{G}^{\mathrm{T} 2{ }_{j_{1}}}$ and an initial column-bus voltage $v_{D}^{\mathrm{T} 4}\left(t_{0}\right)$, known at time $t_{0}$ when switch $\mathrm{T} 3_{j_{1}}$ is closed. When the switch is closed, the column bus will charge or discharge towards the steady-state value [3], i.e.,

$$
V_{D}^{\mathrm{T} 4}=V_{G}^{\mathrm{T} 2}-V_{T}^{\mathrm{T} 2}-\sqrt{\frac{K^{\mathrm{T} 4}}{K^{\mathrm{T} 2}}}\left(V_{G S}^{\mathrm{T} 4}-V_{T}^{\mathrm{T} 4}\right) .
$$

The rate of charging or discharging depends on the load impedance seen by the source follower at this node. Such a load includes the distributed capacitances and resistances of the long metal line on the die connecting all the pixels in the column, as well as the gate capacitance of the input transistor to the next stage. However, on the basis of parameters of a $0.35 \mu \mathrm{m} 3.3 \mathrm{~V}$ AMS process [13], these factors are insignificant compared with the source-bulk capacitances of the $N_{1}$ switches connected to the node, especially as $N_{1}$ is on the order of 1000 for mega-pixel sensors.

Fig. 2 presents a simplification of the circuit in Fig. 1 for the purpose of transient analysis. The load capacitance $C$ is approximated by taking the source-bulk capacitance $C_{\mathrm{SB}}^{\mathrm{T} 3}$ of a switch T3 in parallel $N_{1}$ times, i.e.,

$$
C \approx N_{1} C_{\mathrm{SB}}^{\mathrm{T} 3}
$$

In turn, the source-bulk capacitance approximately equals the depletion capacitance of the reverse biased pn-junction between the source diffusion and bulk substrate of T3, i.e.,

$$
C_{\mathrm{SB}}^{\mathrm{T} 3} \approx \frac{A_{D}^{\mathrm{T} 3} C J}{\left(1+\frac{V_{\mathrm{SB}}^{\mathrm{T}}}{P B}\right)^{M J}}+\frac{P_{D}^{\mathrm{T} 3} C J S W}{\left(1+\frac{V_{\mathrm{SB}}^{\mathrm{T} 3}}{P B}\right)^{M J S W}}
$$

which depends on the area $A_{D}^{\mathrm{T} 3}$ and perimeter $P_{D}^{\mathrm{T}} 3$ of the diffusion (not the same as the area and perimeter of the transistor) and various process parameters $C J, C J S W, M J, M J S W$, and $P B$ [14]. Although $C_{\mathrm{SB}}^{\mathrm{T} 3}$ depends on the source-bulk voltage $V_{\mathrm{SB}}^{\mathrm{T} 3}$, a worst case capacitance may be obtained by setting this voltage equal to zero.

Because the drain of $\mathrm{T} 2$ in Fig. 2 is connected to $V_{D D}$ (and the pixel-drive voltage is never more than $V_{D D}$ ), T2 is always in saturation. On the other hand, T4 is in saturation only if the column-bus voltage is sufficiently high so that

$$
V_{G S}^{\mathrm{T} 4}-V_{T}^{\mathrm{T} 4} \leq V_{D S}^{\mathrm{T} 4}
$$


Normally, the circuit is designed and the column bias $V_{G}^{\mathrm{T} 4}$ is chosen so that, with the switch closed, T4 is in saturation for the expected range of the pixel-drive voltage. However, if the column bus has discharged to ground, as would happen if all switches in Fig. 1 were open, then T4 will be in the linear region for any column bias greater than the threshold voltage. In this region, the transistor behaves like a resistor between the drain and source, with a resistance determined by the gate voltage. As this resistance would be small and in parallel with the load capacitance of Fig. 2, the overall load impedance would be small. Therefore, when the switch is closed, T2 will conduct a current to charge this impedance very quickly and the column-bus voltage will quickly reach a level where T4 enters saturation.

Although it is possible to determine the transient response analytically for the case where $\mathrm{T} 4$ is in the linear region, using Level 1 models and neglecting the output resistance of T2, little of the transient response is affected by assuming T4 is always in saturation regardless of the column-bus voltage. Proceeding with this assumption, using Level 1 models and neglecting the output resistance of $\mathrm{T} 2$ and $\mathrm{T} 4$ in saturation, a differential equation governing the transient response of the circuit is

$$
\begin{aligned}
K^{\mathrm{T} 2}\left(V_{G}^{\mathrm{T} 2}-v_{D}^{\mathrm{T} 4}(t)\right. & \left.-V_{T}^{\mathrm{T} 2}\right)^{2} \\
& =K^{\mathrm{T} 4}\left(V_{G S}^{\mathrm{T} 4}-V_{T}^{\mathrm{T} 4}\right)^{2}+C \frac{\mathrm{d} v_{D}^{\mathrm{T} 4}}{\mathrm{~d} t} .
\end{aligned}
$$

Using standard techniques [15], the solution is

$v_{D}^{\mathrm{T} 4}(t)=V_{G}^{\mathrm{T} 2}-V_{T}^{\mathrm{T} 2}-\sqrt{\frac{K^{\mathrm{T} 4}}{K^{\mathrm{T} 2}}}\left(V_{G S}^{\mathrm{T} 4}-V_{T}^{\mathrm{T} 4}\right) \frac{A(t)+B}{1+A(t) B}$

where

$$
\begin{aligned}
A(t) & =\tanh \left(\frac{\sqrt{K^{\mathrm{T} 2} K^{\mathrm{T} 4}}}{C}\left(V_{G S}^{\mathrm{T} 4}-V_{T}^{\mathrm{T} 4}\right)\left(t-t_{0}\right)\right) \\
B & =\sqrt{\frac{K^{\mathrm{T} 2}}{K^{\mathrm{T} 4}}}\left(\frac{V_{G}^{\mathrm{T} 2}-V_{T}^{\mathrm{T} 2}-v_{D}^{\mathrm{T} 4}\left(t_{0}\right)}{V_{G S}^{\mathrm{T} 4}-V_{T}^{\mathrm{T} 4}}\right) .
\end{aligned}
$$

Although Salama and El Gamal report a similar result for the transient response of the readout circuit in a CMOS image sensor [12], they do not explain the origin of the load capacitance. Moreover, their paper does not concern noise, fixed pattern, or otherwise.

The circuit in Fig. 1 was simulated in Cadence using BSIM3 models of transistors [16] in a $0.35 \mu \mathrm{m} 3.3 \mathrm{~V}$ AMS process [13]. The widths of all transistors were set to $1 \mu \mathrm{m}$, the width of the drain and source contacts, and the lengths to $0.6 \mu \mathrm{m}$, the minimum length recommended by AMS for transistors in analog circuits sensitive to threshold voltage variation [17]. Transient simulations were performed with the column bias $V_{G S}^{\mathrm{T} 4}$ set to $1 \mathrm{~V}$ and the pixel-drive voltage $V_{G}^{\mathrm{T} 2}$ swept from 2.2 to $2.6 \mathrm{~V}$, a range typical of a logarithmic pixel in the process. To simulate uniform illumination on the focal plane, the pixel-drive voltage of the deselected pixels equaled the pixel-drive voltage of the selected pixel. Considering a high-definition television (HDTV) format [11], the number of pixels $N_{1}$ in the column was 1080 .

In addition to the BSIM3 parameters for simulation purposes, AMS provided Level 1 parameters for informational purposes.
These can be used with (7)-(9), where the load capacitance is given by (3) and (4), to approximate the above simulation in MATLAB. Assuming $t_{0}$ and $v_{D}^{\mathrm{T} 4}\left(t_{0}\right)$ are zero, Figs. 3 and 4 show the column-bus voltage $v_{D}^{\mathrm{T} 4}$ as a function of time $t$ and pixeldrive voltage $V_{G}^{T 2}$ for the Level 1 model and BSIM3 simulation, respectively.

The figures have the same trend and show that the column-bus voltage rises to the steady-state value like the step-response of a first-order low-pass filter. The steady-state values of Fig. 4 are lower than those of Fig. 3 because the BSIM3 simulation considers many effects not included in the Level 1 model, such as the body effect of $\mathrm{T} 2$, the on-resistance of $\mathrm{T} 3$ and the finite output resistance of $\mathrm{T} 2$ and $\mathrm{T} 4$ in saturation.

The settling time of the readout circuit in the BSIM3 simulation may be predicted from the Level 1 model. As $t$ increases, $A(t)$ in (8) approaches unity and $v_{D}^{\mathrm{T} 4}(t)$ in (7) approaches the steady-state result in (2). The settling time depends on the value of $B$ in (9), which is a function of the pixel-drive voltage $V_{G}^{\mathrm{T} 2}$ and the initial voltage of the column bus $v_{D}^{\mathrm{T}} 4\left(t_{0}\right)$. However, the closer $A(t)$ gets to unity the less $B$ matters. Thus, the settling time is proportional to the time constant

$$
\tau=\frac{C}{\sqrt{K^{\mathrm{T} 2} K^{\mathrm{T} 4}}} \times \frac{1}{\left(V_{G S}^{\mathrm{T} 4}-V_{T}^{\mathrm{T} 4}\right)}
$$

which equals $40 \mathrm{~ns}$, according to the Level 1 parameters from AMS, and matches the observed settling time in Figs. 3 and 4. Salama and El Gamal discuss further how the settling time depends on design parameters [12], which is not the subject of this paper.

The above derivation gives the transient response of the column-bus voltage for any pixel in a column. The column-bus voltage is switched in turn to drive an output bus, shared by all columns, via a second-stage source follower, which mirrors the parallel source followers of Fig. 1 except with PMOS transistors instead of NMOS transistors [3]. Analysis of the transient response of the output-bus voltage for any column-drive voltage is complementary to the above analysis and, thus, is not repeated. In the case of column-level ADC, data conversion is performed after the first-stage readout described above, which means the second-stage readout is purely digital.

\section{FIXED PATtern NOISE (FPN)}

If sufficient time is allowed between the switching of a row or column in the image sensor and digitization, then the response of the column buffer or the output buffer will settle to the steady-state value. The settling time depends on design parameters of the readout circuit, as well as the initial voltage of the node being charged, i.e., the column bus or the output bus, and the final voltage, as determined by the steady-state equation. For example, if the initial and final voltage were the same, then no charging or discharging need occur and the settling time would be zero. The settling time allowed by the readout controller should be based on the voltage changes that are likely to occur in the camera upon switching from a pixel to its neighbor. This time must accommodate the variety of charging and discharging demands, while meeting the speed requirements of the application. Note that responses of neighboring pixels are normally correlated. 
Care must be taken by the readout controller when the raster scan completes reading the array and begins again at the first row and when the raster scan completes reading the last column of one row and switches to the first column of the next row. Since these jumps involve extra logic processing in the controller, to generate appropriate addressing signals or to encode synchronization bits for display purposes, the column or output bus may have time to discharge. Thus, at the start of every frame scan, the column bus in every column of the array may be required to cover a greater voltage change, than the usual transition from one row to the next, in the usual settling time. Similarly, at the start of every row scan, the output bus may be required to cover a greater voltage change, than the usual transition from one column to the next, in the usual settling time. The demands on the output bus are more critical than those on the column bus as the former must switch about 1000 times faster, i.e., at the pixel scan rate instead of the row scan rate, and may have the initial voltage problem once per row instead of just once per frame.

These problems may be avoided by ensuring there is no greater delay between reading the last pixel in one frame and the first pixel in the next, or between the last pixel in one row and the first pixel in the next, than there is between reading a pixel in the middle of the array and its neighbor. But an egalitarian approach to choosing the settling time implies a low pixel scan rate, one that wastes time when reading the majority of pixels in the array. A better solution would be for the readout controller to permit extra time for settling at every start of a frame or row scan.

If the column-bus or output-bus voltage does not settle prior to digitization, then noise ensues. Consider an array of $N_{1}$ rows and $N_{2}$ columns of pixels, indexed by $j_{1}$ and $j_{2}$, where $1 \leq j_{1} \leq$ $N_{1}$ and $1 \leq j_{2} \leq N_{2}$. It will take $N_{1} T_{1}$ time to read one frame if the time taken to scan a row is $T_{1}$. Let one period $T_{1}$ be given for settling at the outset. If frame scanning begins at time $t_{0}$, then scanning of row $j_{1}$ begins at time $j_{1} T_{1}+t_{0}$. Assume that the voltage of each column bus at time $t_{0}$ is zero. Assume also that circuit parameters $\left(V_{T}, K\right.$ etc.) are the same from pixel-to-pixel and column-to-column so there is no FPN due to device mismatch. Furthermore, assume the sensor is viewing a uniform scene so that pixel-drive voltages $V_{G}^{\mathrm{T} 2 j_{1}}$ are uniform. With these assumptions, the sampled voltage $v_{D}^{\mathrm{T} 4}(t)$ at time $j_{1} T_{1}+t_{0}$, denoted $V_{D j_{1}}^{\mathrm{T} 4}$, on the first column bus is

$$
V_{D j_{1}}^{\mathrm{T} 4}=V_{G}^{\mathrm{T} 2}-V_{T}-\left(V_{G S}^{\mathrm{T} 4}-V_{T}\right) \frac{A_{j_{1}}+B}{1+A_{j_{1}} B}
$$

where

$$
\begin{aligned}
A_{j_{1}} & =\tanh \left(\frac{K}{C}\left(V_{G S}^{\mathrm{T} 4}-V_{T}\right) j_{1} T_{1}\right) \\
B & =\frac{V_{G}^{\mathrm{T} 2}-V_{T}}{V_{G S}^{\mathrm{T} 4}-V_{T}} .
\end{aligned}
$$

Unless $T_{1}$ is sufficiently large so that $A_{j_{1}}$ in (12) approximates unity for $j_{1}=1$, the column-bus voltage $V_{D j_{1}}^{\mathrm{T} 4}$ in (11) will depend on row number $j_{1}$, at least for the first several rows, despite the uniform scene. When $j_{1}$ gets large enough, the column-bus voltage will settle to the steady-state value

$$
V_{D j_{1}}^{\mathrm{T} 4}=V_{G}^{\mathrm{T} 2}-V_{G S}^{\mathrm{T} 4}
$$

While these results were derived for the first column, a similar situation exists for all columns. Thus, even with no spatial variation of device parameters, a row-to-row variation may appear in the digital response of an image sensor due to the transient response of the first-stage readout. A similar and simultaneous column-to-column variation would occur due to insufficient settling time in the second-stage readout when column-bus voltages, indexed by $j_{2}$, are switched in sequence to drive the output bus from a discharged state.

Established methods to calibrate FPN due to random variation of device parameters [3] may accommodate some of the FPN caused by premature digitization. Without transient effects, the relationship between the pixel-drive voltage $V_{G}^{\mathrm{T} 2}$ and the column-bus voltage $V_{D j_{1}}^{\mathrm{T}} 4$ is given by a linear equation, i.e., (14), with constant coefficients from row-to-row. With transient effects, the same relationship may be approximated by linear equations with offsets $a_{j_{1}}$ and gains $b_{j_{1}}$ that vary from row-torow, i.e.,

$$
V_{D j_{1}}^{\mathrm{T} 4}=a_{j_{1}}+b_{j_{1}} V_{G}^{\mathrm{T} 2}
$$

Taking (11) and performing a first-order Taylor expansion of $V_{D j_{1}}^{\mathrm{T} 4}$ in terms of $V_{G}^{\mathrm{T} 2}$ around a reference voltage $\bar{V}_{G}^{\mathrm{T} 2}$ gives the offsets and gains, i.e.,

$$
\begin{aligned}
a_{j_{1}} & =\bar{V}_{G}^{\mathrm{T} 2}\left(1-b_{j_{1}}\right)-V_{T}-\left(V_{G S}^{\mathrm{T} 4}-V_{T}\right) \frac{A_{j_{1}}+B}{1+A_{j_{1}} B} \\
b_{j_{1}} & =1-\frac{1-A_{j_{1}}^{2}}{\left(1+A_{j_{1}} B\right)^{2}}
\end{aligned}
$$

where

$$
\begin{aligned}
A_{j_{1}} & =\tanh \left(\frac{K}{C}\left(V_{G S}^{\mathrm{T} 4}-V_{T}\right) j_{1} T_{1}\right) \\
B & =\frac{\bar{V}_{G}^{\mathrm{T} 2}-V_{T}}{V_{G S}^{\mathrm{T} 4}-V_{T}} .
\end{aligned}
$$

Returning to the HDTV example of Section II, note that in Figs. 3 and 4 the relationship between the pixel-drive voltage and column-bus voltage at any time $t$, or $j_{1} T_{1}$ when $t_{0}$ is zero, approximates a straight line. However, the intercept and slope of the lines, called the offset and gain in the context of readout, vary with time. They are plotted in Fig. 5.

The time-varying coefficients were calculated using (16) and (17) for Fig. 3, where $\bar{V}_{G}^{\mathrm{T} 2}=2.4 \mathrm{~V}$, and using linear regression for Fig. 4. For a small sampling period $T_{1}$, the figure shows that the offset and gain vary for small row numbers $j_{1}$ but eventually settle.

The modeling and simulation results generally agree and show that a variation in the offset and gain of the source follower would occur from row-to-row if insufficient time was allowed for the column-bus voltage to settle, especially when 


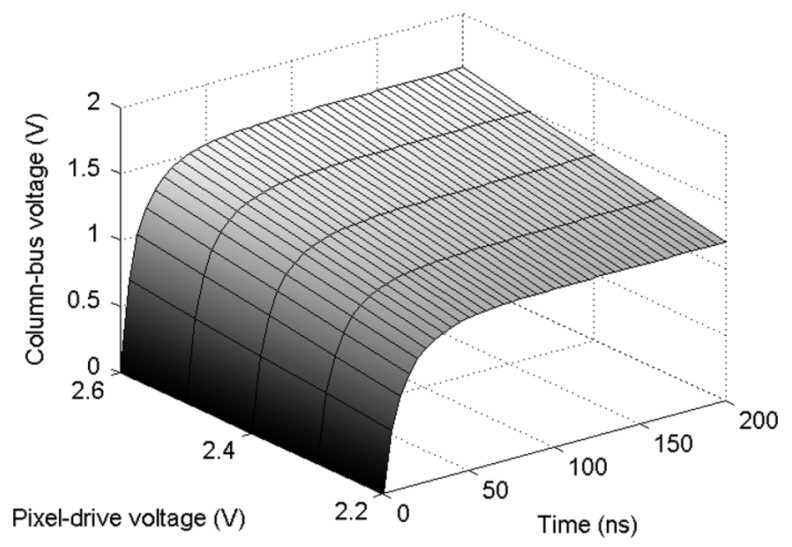

Fig. 3. The transient response of the first-stage readout from a discharged state, using a Level 1 model. Note that, for any time $t$, the column-bus voltage $v_{D}^{\mathrm{T} 4}$ is a linear function of the pixel-drive voltage $V_{G}^{\mathrm{T}} 2$.

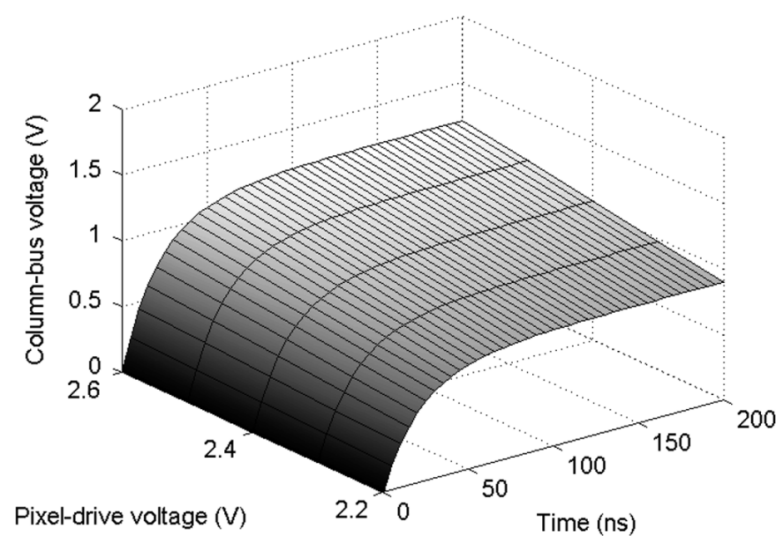

Fig. 4. The transient response of the first-stage readout from a discharged state, using a BSIM3 simulation. Note that, for any time $t$, the column-bus voltage $v_{D}^{\mathrm{T} 4}$ is a linear function of the pixel-drive voltage $V_{G}^{\mathrm{T}} 2$

it begins from a discharged state as may happen at the start of reading each frame. If sufficient time was allowed for the column bus to charge, then the offset and gain of the linear relationship between $V_{G}^{\mathrm{T} 2}$ and $V_{D}^{\mathrm{T} 4}$ would be constant for all rows. In Fig. 5, the coefficients derived from the simulation are smaller in magnitude than those derived from the modeling because of the greater accuracy of the simulation. The modeling has been provided because it explains the trend.

Noting (11)-(13), the relationship between the pixel-drive voltage and the column-bus voltage is not perfectly linear. In other words, a first-order Taylor expansion of (11), as in (15), is only an approximation. The higher order terms of the Taylor expansion are expected to vary from row-to-row, which would cause a slightly nonlinear FPN in the first few rows. By giving extra settling time at the start of reading each frame, transient-induced FPN may be vastly reduced.

Actually, the period $T_{1}$ is large enough in this example for the first-stage readout to settle. The row scan time is about $31 \mu \mathrm{s}$ for an HDTV camera with $1080 \times 1920$ pixels and a frame rate of $30 \mathrm{~Hz}$. Therefore, the column-bus voltage will settle before the first row is read. If scanning of the first row begins as soon as the first row is selected, rather than waiting for one period as assumed above, some pixels in the first row will suffer from
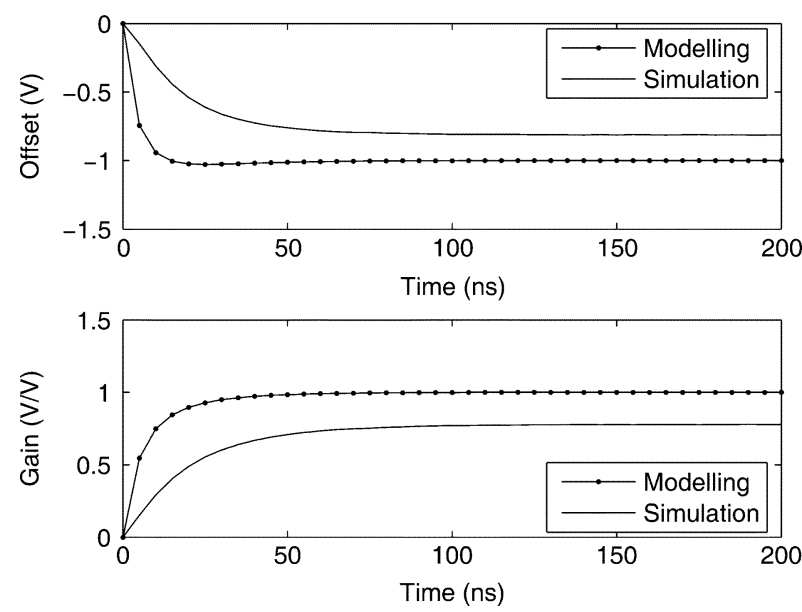

Fig. 5. The offset and gain of the first-stage readout, for the example in Section II, that relates the column-bus voltage $v_{D}^{\mathrm{T} 4}$ linearly to the pixel-drive voltage $V_{G}^{\mathrm{T} 2}$ as a function of time $t$. These plots also give the offset $a_{j_{1}}$ and gain $b_{j_{1}}$ versus row number $j_{1}$, where $j_{1} T_{1}$ is the time at which row $j_{1}$ is sampled.

insufficient settling time though the effect will disappear by the second row. Thus, a row-to-row variation of pixel responses due to the transient response is unlikely. However, some column-tocolumn variation is likely because the period $T_{2}$, representing the time taken by the second-stage readout to switch columns is not large. For the HDTV example, this period is about $16 \mathrm{~ns}$, a fraction of the settling time observed in Fig. 5.

\section{LOGARITHMic CMOS IMAGE SENSORS}

Experiments were conducted using a $512 \times 512$ pixel Fuga 15RGB logarithmic camera, described in detail by Joseph [15]. This imager is a color version of the Fuga 15d, where pixels are overlaid in columnwise fashion with red, green, and blue filters. As the literature already explains color in logarithmic cameras [4], the Fuga 15RGB is treated here as if it were monochromatic. Results presented in this section have been filtered columnwise by a three-point moving-average filter to cancel the variation introduced by the color filters. Such an operation does not prejudice the results but facilitates explanation by avoiding unnecessary detail and qualification.

Although the Fuga 15RGB, interfaced to a computer by a PCI card, was capable of a full frame rate of about $8 \mathrm{~Hz}$ [18], images were very noisy at this speed. Workable performance could be achieved only below $4 \mathrm{~Hz}$. The camera offered four timing settings to the programmer called the $\mathrm{X} 1, \mathrm{X} 2, \mathrm{Y}$, and ADC delays [19]. The X1 delay controlled the time permitted for settling after a change in the column number (or X-address). This setting had the greatest effect on the speed and image, apart from the ADC delay, and was used to control the frame rate. For reasons that remain unclear, the $\mathrm{X} 2$ delay provided an extra delay every 32 nd column of the raster scan. However, this setting had almost no effect on the speed or image and was set to the maximum value. The Y delay, possibly a feature not fully implemented in the device driver, had no effect though it reportedly controlled the time permitted for settling after a change in the row number (or Y-address). Finally, the ADC delay controlled the time permitted for settling at the ADC input and was set to the maximum value. Above a critical value, the setting had little 


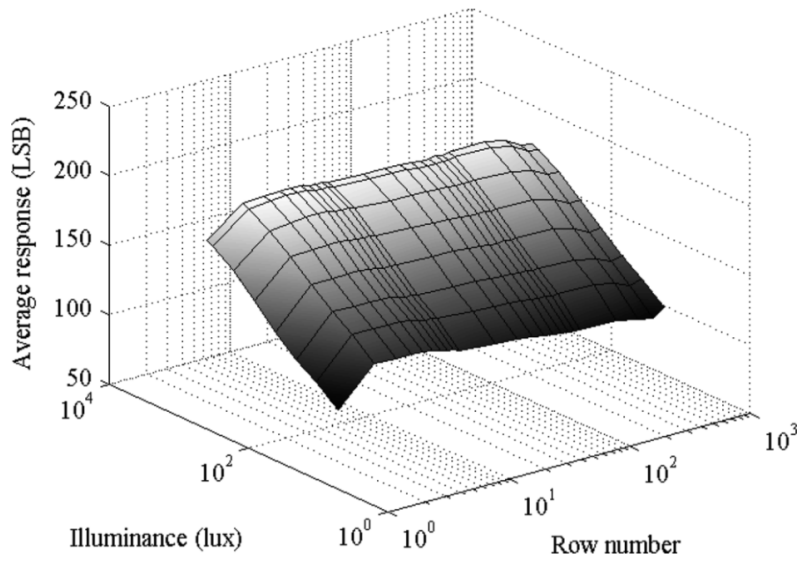

Fig. 6. The average response $\bar{y}_{i j_{1}}$ of each row of the Fuga 15RGB versus illuminance $x_{i}$ and row number $j_{1}$. For any row, the average response depends linearly on the logarithm of illuminance. Each row number corresponds to the time the row is digitized from the start of frame scanning by the first-stage readout.

effect on overall speed or image quality but, below this value, both speed and noise increased sharply.

After setting the X1 delay, which was an integer between 0 and 255 , the frame rate was computed by measuring on the computer the time taken between readout of consecutive frames. Some variability existed in this measurement as the multitasking operating system made use of preemptive scheduling but it was compensated for with a moving average filter, a fast processor and by not running other applications in the background. Images were taken of a sheet of white paper, under fluorescent office lighting, to provide a uniform scene. The aperture setting of the lens was varied to simulate intensity variation of the illuminant.

Eight images were taken of the white paper, varying the aperture from 1.8 to 16 f-stops to simulate a two-decade intensity variation of the illuminant. These images were captured at a frame rate of $0.45 \mathrm{~Hz}$, the slowest possible speed of the Fuga 15RGB. The pixel responses, denoted $y_{i j_{1} j_{2}}$, where $i$ ranges over the images $(1 \leq i \leq 8), j_{1}$ ranges over the rows $\left(1 \leq j_{1} \leq\right.$ $512)$ and $j_{2}$ ranges over the columns $\left(1 \leq j_{2} \leq 512\right)$, were averaged over the columns and rows, respectively, to give rowwise and columnwise profiles $\bar{y}_{i j_{1}}$ and $\bar{y}_{i j_{2}}$, respectively, i.e.,

$$
\begin{aligned}
& \bar{y}_{i j_{1}}=\frac{1}{N_{2}} \sum_{j_{2}=1}^{N_{2}} y_{i j_{1} j_{2}} \\
& \bar{y}_{i j_{2}}=\frac{1}{N_{1}} \sum_{j_{1}=1}^{N_{1}} y_{i j_{1} j_{2}} .
\end{aligned}
$$

Fig. 6 shows the average response $\bar{y}_{i j_{1}}$ of each row versus illuminance $x_{i}$, calculated using the f-stop settings and the measured illuminance of the paper, and row number $j_{1}$.

The row number, which is proportional to the time the row was read after the start of reading each frame, is on a logarithmic scale to highlight the first few rows, while showing all rows. To avoid cluttering the plot with too many lines, as there were

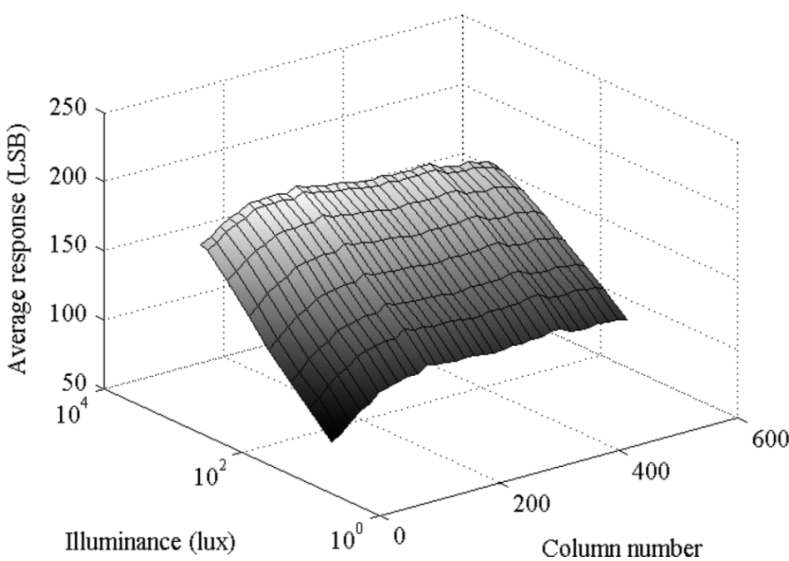

Fig. 7. The average response $\bar{y}_{i j_{2}}$ of each column of the Fuga 15RGB versus illuminance $x_{i}$ and column number $j_{2}$. For any column, the average response depends linearly on the logarithm of illuminance. Each column number corresponds to the time the column is digitized from the start of row scanning by the second-stage readout.

512 rows, responses were averaged rowwise in exponentially increasing bins. ${ }^{1}$ The figure shows an insufficient settling time for the first-stage readout. Unlike in Figs. 3 and 4, responses of the first row in Fig. 6 depend on illuminance rather than equal a constant value (zero in Figs. 3 and 4), which means the Fuga 15RGB gave some settling time from the initial condition though not quite enough.

Fig. 7 shows the average response $\bar{y}_{i j_{2}}$ of each column versus illuminance $x_{i}$ and column number $j_{2}$, which is proportional to the time the column was read after the start of reading each row.

To avoid cluttering the plot with too many lines, as there were 512 columns, responses were averaged columnwise in bins of 16 columns. The figure shows an insufficient settling time for the second-stage readout, as illustrated in Figs. 3 and 4, that affects many columns. Similar to the first row in Fig. 6, responses in the first column of Fig. 7 depend on illuminance because the Fuga 15RGB gave some settling time from the initial condition. However, especially since the camera was operated at the slowest speed, this time was vastly insufficient.

For any row number in Fig. 6 and any column number in Fig. 7, there is an approximate linear relationship between the average response and the logarithm of illuminance. As with the modeling and simulation results, the gain and offset of this linear dependence vary in a continuous manner, as opposed to a random manner if there was only steady-state FPN. The lack of surface smoothness in these figures, as compared with Figs. 3 and 4 , indicates the presence of device mismatch $\left(V_{T}, K\right.$, etc.), a random variation that has been attenuated by the averaging.

The triple variation model, summarized in Section II, was calibrated to the data $y_{i j_{1} j_{2}}$ [3]. Estimated offsets $\hat{a}_{j_{1} j_{2}}$, gains $\hat{b}_{j_{1} j_{2}}$ and biases $\hat{c}_{j_{1} j_{2}}$ were averaged rowwise and columnwise. The procedure was repeated for frame rates of 1.50 and $2.51 \mathrm{~Hz}$. Parameter profiles are plotted in Fig. 8 with the row number on a logarithmic scale but no binning across rows or columns.

${ }^{1}$ Responses in rows 1-9 were not averaged, whereas responses in rows $10-99,100-499$, and 500-512 were averaged in bins of 10, 100, and 13 rows, respectively. 

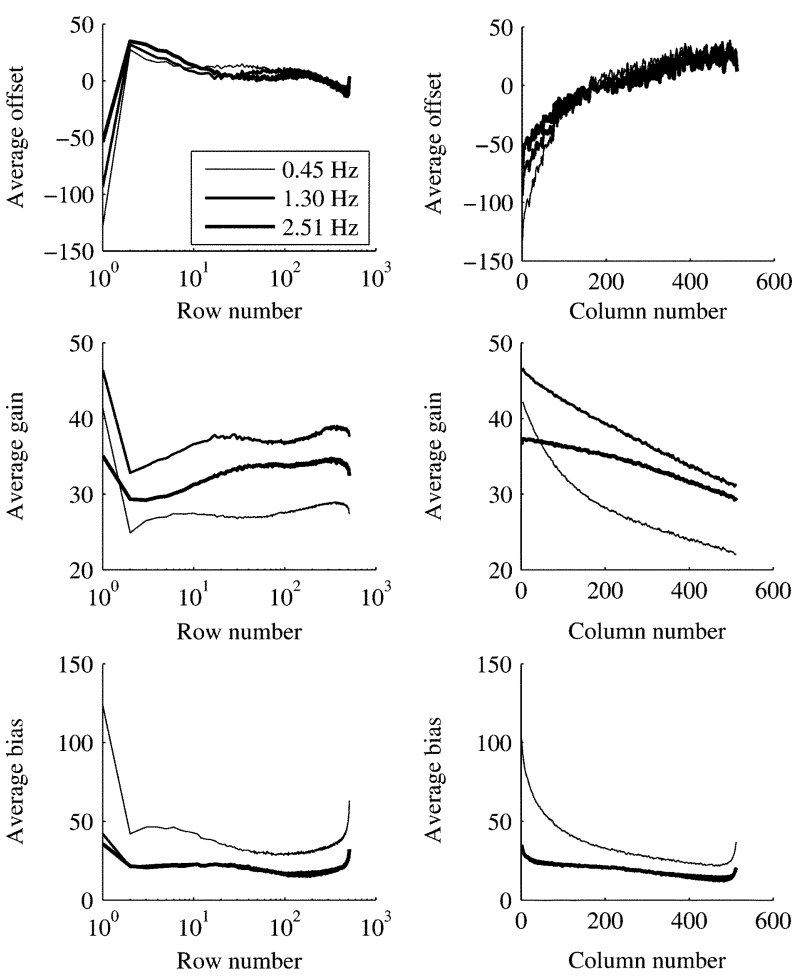

Fig. 8. The average offset, gain, and bias of each row and column of the Fuga $15 \mathrm{RGB}$, after calibration of the triple variation model [3], for frame rates of $0.45,1.30$, and $2.51 \mathrm{~Hz}$. The row or column number corresponds, respectively, to the time at which the row or column is digitized since the start of a frame or row scan.

The offset and gain profiles have somewhat similar trends to the modeling and simulation results of Fig. 5. Unfortunately, the makers of the Fuga 15RGB gave few details on their particular readout circuit. For this reason, the analysis of Section II was based on a readily-available $0.35 \mu \mathrm{m} 3.3 \mathrm{~V}$ process [13] and a well-known readout circuit [20], both of which may not match the actual design.

For simplicity, the modeling and simulation results ignored bias variation. Calibration of this variation accommodates some of the transient response, as shown in Fig. 8, and affects the dependence of the estimated offset and gain on row or column number. Note that the bias profile is basin shaped, rowwise, and columnwise, which is consistent with vignetting-an optical variation due to aperture effects that has been modeled in the literature [3]. The bias would be higher at the borders because photocurrents would be smaller there relative to leakage currents. Note also that the parameter profiles change as the frame rate changes, which implies a transient dependence. The columnwise offset profiles have the simplest dependence on frame rate, settling more steeply for slow rates than for fast rates.

The offset, gain, and bias parameters, which govern pixel responses to light stimuli, depend on the readout speed of the Fuga 15RGB. Consequently, if the camera is calibrated at one speed and estimated parameters are used to correct FPN at another speed, there will be a residual error. The simplest way to calibrate and correct FPN is to subtract the image of a uniform scene from subsequent images, a method that at best may correct offset variation. Such a feature is built into the Fuga 15RGB and, without explanation, the makers warn that recalibration is

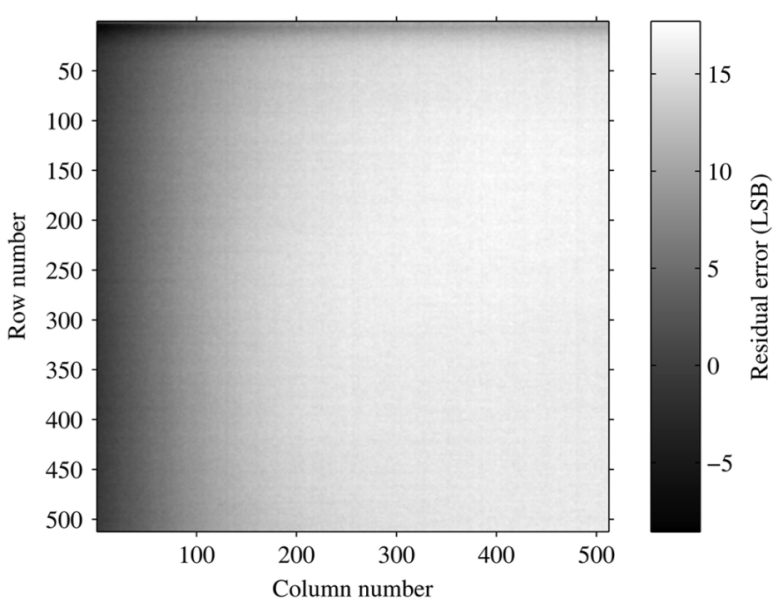

Fig. 9. The difference between two Fuga 15RGB images (fast minus slow) of a uniform scene taken at frame rates of 0.45 and $1.30 \mathrm{~Hz}$ with a fully open aperture. Dead pixels were removed by median filtering [15]. The greatest variation in this difference occurs in the first 100 to 200 columns and the first few rows.

necessary if the readout delays are changed [19]. For example, Fig. 9 shows the difference between two images of the same uniform scene taken at a slow and fast frame rate.

The "fast" image deviates significantly from the "slow" image. As expected, the difference is neither uniform nor random but is strongly and weakly a function of the column and row number, respectively. Considering Sections II and III, one expects the "fast" and "slow" images to have a difference of zero at high column and row numbers. This does not occur with the Fuga 15RGB, implying that its actual readout circuit, which remains unpublished, has complexities that have not been considered here.

Although Figs. 8 and 9 show that the transient response of the readout circuit causes FPN, they do not measure it relative to the FPN caused by the steady-state response of the mismatched pixels. While there are several ways to demonstrate that the gain variation of the Fuga 15RGB is primarily due to premature digitization, an established hypothesis-testing approach [3] has been adopted here. In addition to the triple variation model, one may propose "single" and "double variation" models to describe a logarithmic imager. With single variation, only the offset may vary from one pixel to another. With double variation, the offset and gain may vary spatially. One tests the three hypotheses by calibrating each model and checking the residual error. In the single and double variation cases, the residual error has been shown to depend strongly on illuminance, contradicting the assumption that $\epsilon$ in (1) was statistically independent of $x$.

For the frame rate of $0.45 \mathrm{~Hz}$, the single, double, and triple variation models were calibrated to the data $y_{i j_{1} j_{2}}$. A new hypothesis was also tested. In the "constrained triple variation" model, the offset and bias may vary from one pixel to another but the gain may only vary from one column to another, i.e., the gain was constrained to be constant in each column of 512 pixels. The residual errors versus illuminance, plotted in Fig. 10, show that the performance of constrained and unconstrained triple variation is comparable.

In either case, the residual errors are less than 1 LSB and are mostly independent of illuminance. However, the constrained 


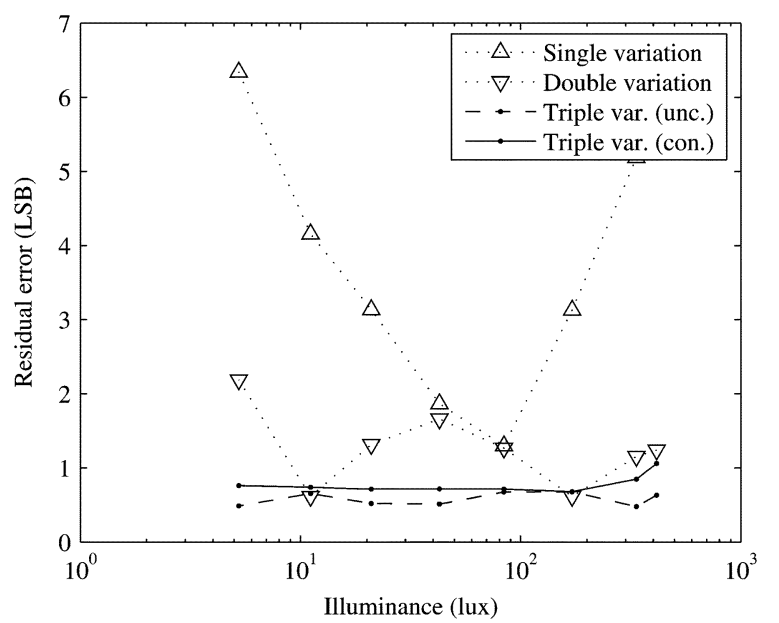

Fig. 10. The residual error per image $\hat{\sigma}_{\epsilon_{i}}$, as defined in the literature [3], versus illuminance $x_{i}$ for calibration of the triple variation models, unconstrained and constrained, to Fuga 15RGB responses. Results for the single and double variation models are included for comparison.

model has $2+(1 / 512)$ free parameters per pixel, almost the same as double variation. With three free parameters per pixel, the unconstrained triple variation model is overly general. Thus, over a two-decade dynamic range, the gain variation observed in the Fuga 15RGB is almost entirely a columnwise variation with a nonrandom profile. Insufficient settling time in the first-stage readout does introduce a rowwise variation to the gain but it affects a few rows mainly and is therefore negligible. Although not tested above, constraining either the offset or bias in the triple variation model to vary only from one column to another proves to be a poor hypothesis [15]. Hence, steady-state gain variation is small, while offset and bias variation contain a big device-mismatch component.

\section{CONCLUSION}

Whereas the literature explains how parameter variation from pixel-to-pixel affects the steady-state response of a logarithmic camera so as to produce FPN [3], this paper explains in detail how the transient response of the readout circuit may cause FPN regardless of device mismatch. When pixels are scanned at high speed for digitization, the readout circuit must switch very quickly, which makes its transient response crucial to image quality. If a two-stage process is employed before data conversion, one to copy all pixel voltages in a row to column buffers and the other to copy a column-buffer voltage to an output buffer, each stage has different demands on its transient response. The second stage must operate about a thousand times faster than the first stage and is hence more critical. In the case of column-level data conversion, only the first stage may contribute to FPN and it will do so at very high frame rates.

A model of the transient response of the readout circuit, typical for both the first and second stage, was constructed by solving a differential equation relating the input and output voltage of a switched source follower to the designable parameters of the circuit and the initial voltage of the output. When one row or one column is selected in the first or second stage, all other rows or columns have open switches, which are transistors in the cutoff region. The model identifies the load impedance of the readout circuit to be the parallel combination of the source-bulk capacitances of all the switch transistors. This was confirmed with a simulator. These results apply to CMOS processes in which transistors may be coarsely and finely approximated by Level 1 and BSIM3 models, respectively. For deep-submicron CMOS processes $(0.13 \mu \mathrm{m}$ and below), the analysis presented here will need to be refined further.

Modeling and simulation were used to show that if the readout circuit does not permit adequate settling time, then digitized responses will vary in a predictable manner from row-to-row or from column-to-column of an image sensor, even with a uniform stimulus and no device mismatch. Furthermore, these effects would appear principally as an offset and gain variation correlated to the row or column number, as opposed to a purely random offset and gain variation. Such variation could be calibrated partly using previously published steady-state methods. The effects would be most noticeable, and hence settling time would be most important, for the topmost rows or the leftmost columns of the image sensor as the greatest voltage changes are likely to occur at the outset of each frame or row scan.

Experiments were performed with a Fuga 15RGB logarithmic camera. Images were taken of uniform scenes with different aperture settings of the lens, to simulate illuminance variation, and with different speed settings of the readout circuit. The results demonstrated substantial variation of digital responses in a predictable manner that echoed the modeling and simulation. Digital responses were calibrated using the established triple variation model of FPN [3]. Profile plots were used to show how the offset and gain depended on the transient response. The bias also depended on the transient response but showed signs of vignetting. Transient effects were more significant across columns than across rows. Calibration of the triple variation model with a constraint on the gain so that it could vary only from one column to another gave a residual error very similar to the case of unconstrained triple variation. The sum-total of the evidence proved that the majority of the gain variation was due to transient rather than steady-state causes.

Like steady-state effects due to device mismatch, transient effects due to premature digitization may be a significant cause of FPN in logarithmic CMOS image sensors. Although much of this effect may be calibrated by assuming offset and gain variation, due to the flexibility of the steady-state models to accommodate transient effects, the transient effects are inherently complex and may require digital filtering for proper compensation. The best solution, therefore, is to permit adequate time for settling at the start of reading each frame and row.

\section{REFERENCES}

[1] O. Yadid-Pecht, "Wide-dynamic-range sensors," Opt. Eng., vol. 38, no. 10, pp. 1650-60, Oct. 1999.

[2] Vision for Machines, "One megapixel CMOS stop action camera family," Tech. Rep., DALSA, Feb. 2004.

[3] D. Joseph and S. Collins, "Modeling, calibration, and correction of nonlinear illumination-dependent fixed pattern noise in logarithmic CMOS image sensors," IEEE Trans. Instrum. Measure., vol. 51, no. 5, pp. 996-1001, Oct. 2002.

[4] D. Joseph and S. Collins, "Modeling, calibration, and rendition of color logarithmic CMOS image sensors," IEEE Trans. Instrum. Measure., vol. 52 , no. 5, pp. 1581-7, Oct. 2003. 
[5] B. Choubey, S. Aoyama, S. Otim, D. Joseph, and S. Collins, "An electronic-calibration scheme for logarithmic CMOS pixels," IEEE Sensors J., vol. 6, no. 4, pp. 950-6, Aug. 2006.

[6] S. Otim, B. Choubey, D. Joseph, and S. Collins, "Model based fixed pattern noise correction for high dynamic range logarithmic CMOS imagers," IEEE Trans. Instrum. Measure., 2006, accepted.

[7] G. Storm, J. E. D. Hurwitz, D. Renshaw, K. Findlater, R. Henderson, and M. Purcell, "High dynamic range imaging using combined linearlogarithmic responses from a CMOS image sensor," in Proc. IEEE Workshop on Charge Coupled Devices and Advanced Image Sensors, May 2003, pp. 1-6.

[8] G. Storm, R. Henderson, J. E. D. Hurwitz, D. Renshaw, K. Findlater, and M. Purcell, "Extended dynamic range from a combined linear-logarithmic CMOS image sensor," IEEE J. Solid-State Circuits, vol. 41, no. 9, pp. 2095-106, Sep. 2006.

[9] M. Tabet, N. Tu, and R. Hornsey, "Modeling and characterization of logarithmic complementary metal-oxide-semiconductor active pixel sensors," J. Vacuum Sci. Technol. A, vol. 18, no. 3, pp. 1006-9, May-Jun. 2000.

[10] IMS Chips, "HDRC VGA imager and camera data and features," Inst. Microelectronics, Stuttgart, Germany, Tech. Rep., Sep. 2000.

[11] A. C. Luther, Video Camera Technology. Boston, MA: Artech House, 1998.

[12] K. Salama and A. El Gamal, "Analysis of active pixel sensor readout circuit," IEEE Trans. Circuits and Systems-I: Fundamental Theory and Applications, vol. 50, no. 7, pp. 941-4, Jul. 2003.

[13] Austria Micro Systems, " $0.35 \mu \mathrm{m}$ CMOS process parameters," Tech. Rep. 9933016.

[14] K. R. Laker and W. M. C. Sansen, Design of Analog Integrated Circuits and Systems. New York: McGraw-Hill, 1994.

[15] D. Joseph, "Modelling and calibration of logarithmic CMOS image sensors," Ph.D. dissertation, Univ. Oxford, Oxford, U.K., Sep. 30, 2002.

[16] D. P. Foty, MOSFET Modeling with SPICE: Principles and Practise. Upper Saddle River, NJ: Prentice-Hall, 1997.

[17] Austria Micro Systems, "0.35 $\mu \mathrm{m}$ CMOS design rules," Tech. Rep. 9931032.
[18] C-Cam Technologies, "Fuga data sheets," Vector International, Tech. Rep., Apr. 3, 1998,

[19] C-Cam Technologies, "Introduction software for Fuga RGB," Vector International, Apr. 30, 1998.

[20] S. K. Mendis, S. E. Kemeny, R. C. Gee, B. Pain, C. O. Staller, Q. Kim, and E. R. Fossum, "CMOS active pixel image sensors for highly integrated imaging systems," IEEE J. Solid-State Circuits, vol. 32, no. 2 , pp. 187-97, Feb. 1997.

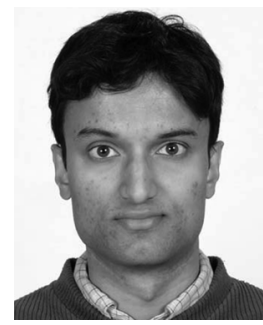

Dileepan Joseph (M'96) received the B.Sc. degree in computer engineering from the University of Manitoba, Winnipeg, MB, Canada, in 1997 and the D.Phil. degree in engineering science from the University of Oxford, Oxford, U.K., in 2002. His doctoral research concerned logarithmic CMOS image sensors.

In 2004, he joined the Faculty of Engineering at the University of Alberta, Edmonton, AB, Canada, where he has continued to work on imaging science.

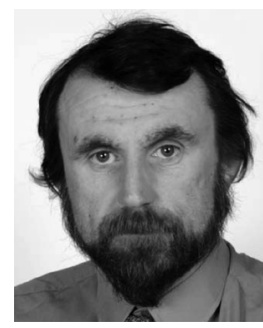

Steve Collins (M'03) received the B.Sc. degree in theoretical physics from the University of York, York, U.K., in 1982 and the Ph.D. degree from the University of Warwick, Warwick, U.K., in 1986.

From 1985 to 1997 , he worked for the Defence Research Agency, Malvern, U.K, on various topics including the origins of $1 / \mathrm{f}$ noise in MOSFETs and analog information processing. Since 1997, he has been with the University of Oxford, Oxford, U.K., where he has continued his interest in smart imaging sensors and nonvolatile analogue memories. 